\title{
Oculokinetic perimetry: a simple visual field test for use in the community
}

\author{
BERTIL E DAMATO \\ From the Tennent Institute of Ophthalmology, University of Glasgow
}

SUMmaRY A method of visual field examination is described which enables an unsupervised person to carry out self-assessment using only a paper test chart, a record sheet, and a pencil. It is entitled 'oculokinetic perimetry' because it is the subject's eye that moves and not the test target. By providing non-ophthalmic health care workers with a simple means of performing perimetry in the community, and by allowing susceptible people to carry out self-assessment of the visual fields at home, this test should facilitate the detection and management of glaucoma, especially in underdeveloped countries.

\begin{abstract}
Visual field examination has an invaluable role in the diagnosis and management of glaucoma and other important ocular and neurological disorders.' For this reason remarkable technological advances have recently taken place in perimetry ${ }^{2}$ in order to improve results. However, these developments have done little to facilitate the detection and monitoring of visual field defects outside ophthalmic clinics. At present examination of the visual fields to confrontation remains the sole means available to many physicians, and with few exceptions ${ }^{3}$ self-assessment of the visual fields at home is of benefit only to patients who have paracentral visual defects. ${ }^{4}$ These limitations occur because conventional methods of perimetry (whereby one or more test stimuli are introduced into the peripheral visual field of an immobile eye) require a highly trained observer and/or expensive equipment $(a)$ to move the test target through all areas of the visual field, $(b)$ to ensure that the subject's eye remains immobile throughout the examination, and (c) to plot the results. The author has attempted to overcome these three obstacles to more universal perimetry by developing an alternative method of visual field examination which is entitled 'oculokinetic perimetry' because it is the subject's eye that is moved and not the test stimulus.
\end{abstract}

Correspondence to $\operatorname{Dr} B$ E Damato, Tennent Institute of Ophthalmology, Western Infirmary, Glasgow G11 6NT.

\section{Material and methods}

OCULOKINETIC VISUAL FIELD TEST

The test chart consists of a white sheet of paper with 100 numbers distributed around a central test stimulus in such a manner as to increase the chances of detecting arcuate, quadrantic, hemianopic, and altitudinal visual field defects as well as a nasal step (Fig. 1).

The numbers are printed in an unsaturated blue colour to avoid confusion with the test stimulus, which is coloured black so that it can if necessary be easily enlarged with an ordinary pen or pencil. The size of the test stimulus $(1.4 / 333.3 \mathrm{~mm})$ subtends a visual angle of $0.25^{\circ}$, so that it is barely visible to normal persons at $25^{\circ}$ from fixation. The record sheet comprises two small versions of the test chart with a set of instructions on the reverse side.

The test is carried out with the test chart attached to a well-lit vertical surface, its centre being placed at eye level. The right eye is tested first so that, soon after starting the examination, the subject is reassured by the detection of the blind spot that the correct procedure is being followed. The correct working distance is found by looking down at the star near the lower border of the chart, covering one eye, and moving backwards or forwards until the letter $\mathbf{L}$ or $\mathbf{R}$ (for the left and right eye respectively) is no longer visible. The numbers are then read slowly from 1 to 100 so that the test target moves through all 


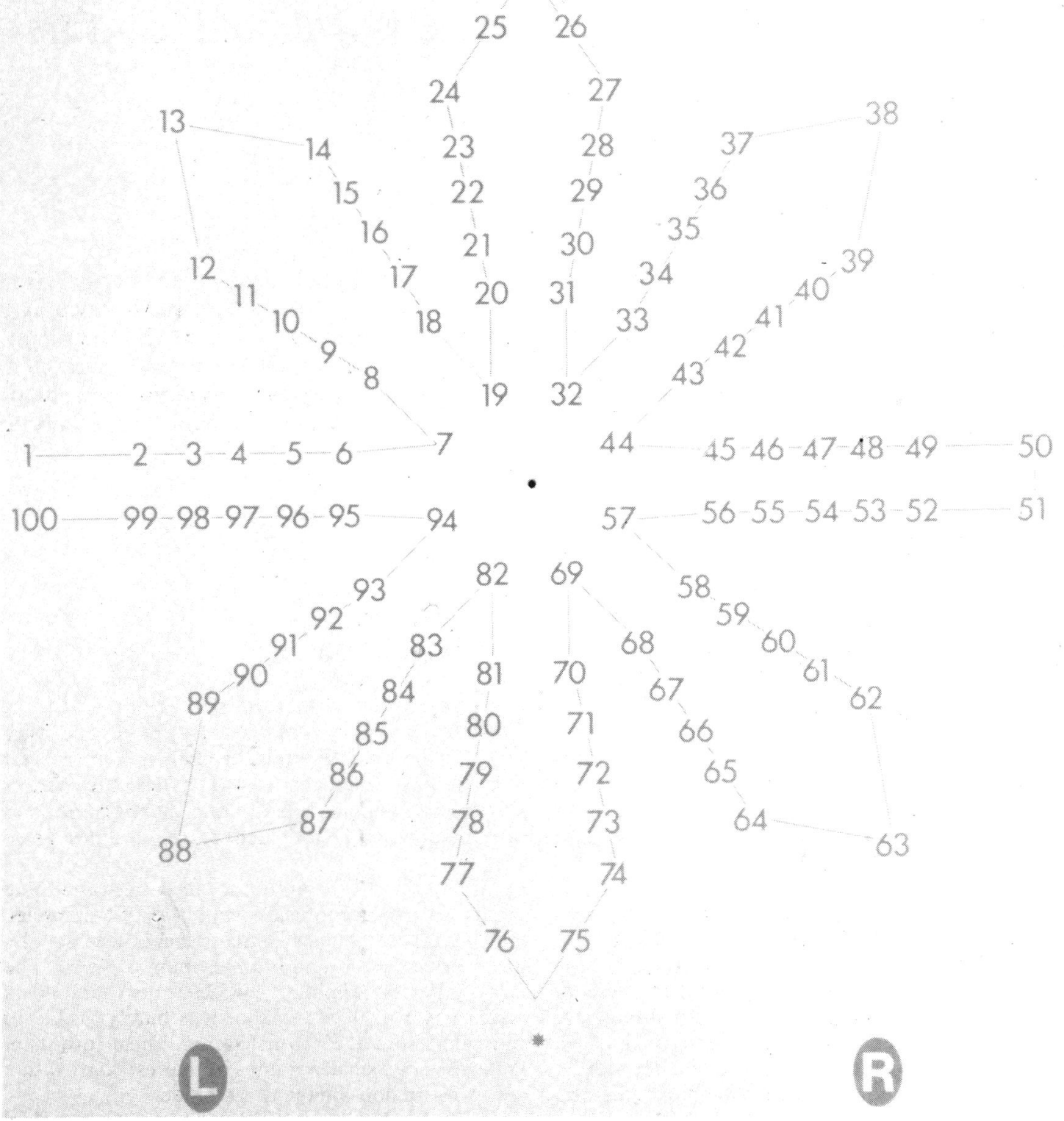

Fig. 1 Oculokinetic visual field test chart. The reader can perform the test (albeit with difficulty because this modified chart is half the size of the real test chart) by holding this page $15 \mathrm{~cm}$ from the eye and using the appropriate reading correction.

areas of the visual field from 5 to $25^{\circ}$. The numbers associated with disappearance of the central test stimulus are noted directly on the record sheet by crossing out the respective number. If a unilateral central scotoma is present, the subject is advised to look at each number with the other eye and then to cover that eye to determine whether the test stimulus can be seen by the affected eye. The results recorded by the subject can subsequently be evaluated by a qualified observer. The completed record sheet must be inverted before the results can be compared with visual fields plotted by conventional techniques. As in all visual field tests, the blind spot is a useful guide to the validity of the examination. 


\section{METHODS}

Over 30 patients attending the Tennent Institute and a peripheral hospital who were known to have visual field defects and 30 healthy volunteers were tested by oculokinetic perimetry. In most cases the examination was performed with the assistance of an optician or a nurse. A few patients had the test chart mailed to their homes, and the results were also returned by mail.

All subjects were encouraged to take their time, to recheck any areas they were unsure of, and to take a break whenever they felt this was required. The results were subsequently assessed in relation to records obtained by opticians using conventional methods.

\section{Results}

The majority of persons invited to carry out oculokinetic perimetry understood what was required of them and were able to complete the examination provided the numbers could be read with one eye.

Three out of 33 patients who seemed to carry out the procedure correctly produced false negative results with oculokinetic perimetry. In these three cases a visual field defect was detected only with the Oculus Tubinger perimeter by using a white target of size 1.0 and a filter setting of 10 (which is approxi- mately equivalent to a Goldmann target size of 1-2e). Good topographic correspondence was obtained in 25 out of the remaining 30 cases (Figs. 2A, B).

Five out of 30 healthy controls missed one or both blind spots. Ten of the remaining 25 persons missed the test stimulus at one or more points when it was situated more than $17 \cdot 5^{\circ}$ from fixation. Although an additional seven persons missed the test stimulus within the central $17 \cdot 5^{\circ}$ of vision, only four of these produced a false positive result. Surprisingly two of these four subjects also produced a positive result with the Tubinger perimeter, one being found to have a congenital abnormality of the optic discs. One subject had attempted to read the numbers while looking at the central target.

Some patients were disturbed by the reading segment in their bifocal spectacles. It is interesting that one patient who had previously been tested by conventional methods became aware of her extensive visual field defect only when she carried out oculokinetic perimetry. Some persons asked if they could obtain disposable visual field test charts to enable them or members of their family to test themselves at home. When a patient with serpiginous choroiditis was informed that this was not possible without her consultant's permission, she immediately decided to draw her own test chart.

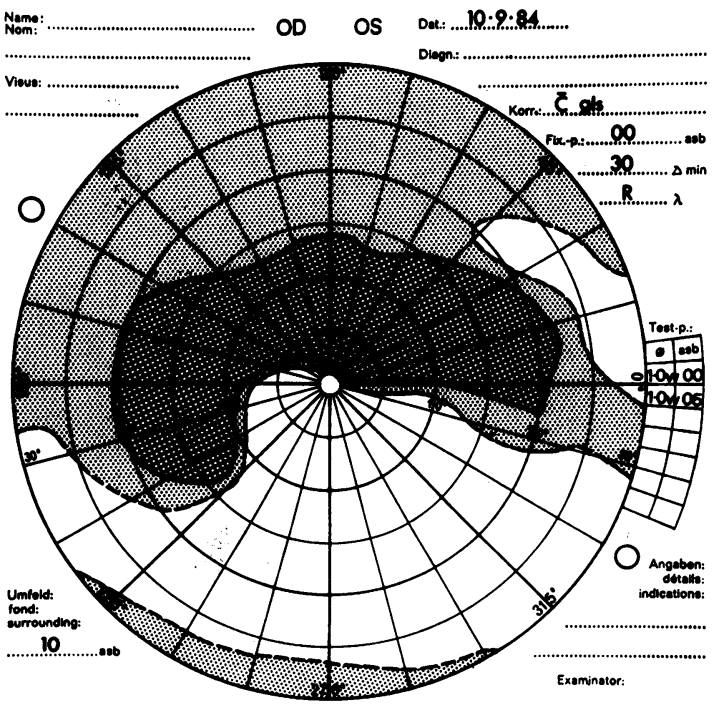

Fig. 2A

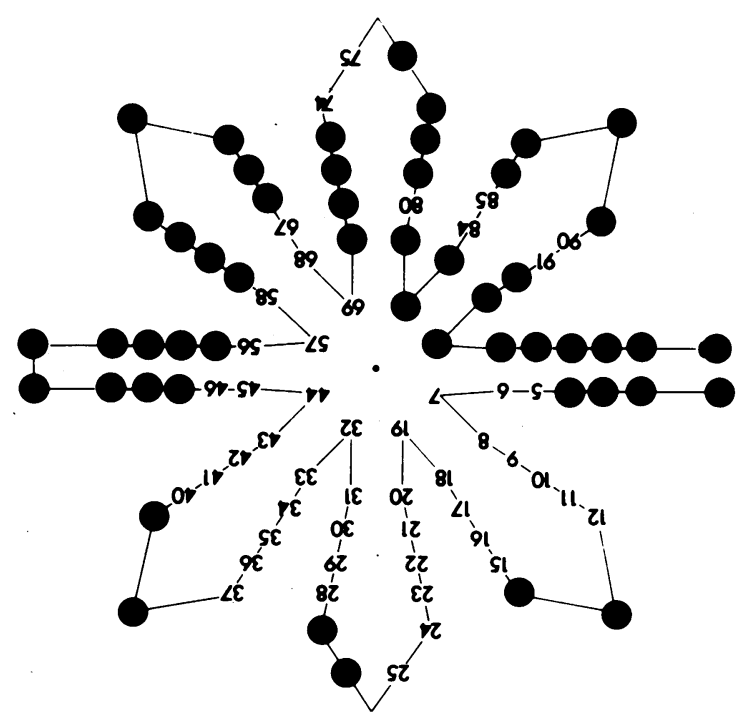

Fig. 2B

Fig. 2 The right central visual field of a 71-year-old female patient with primary open-angle glaucoma plotted (A) with the Tubinger perimeter and (B) by oculokinetic perimetry. A disposable test chart was mailed to the patient's home, and the completed record sheet was returned a week later. 


\section{Discussion}

By moving the eye instead of the test stimulus it is possible for an unsupervised person to carry out selfassessment of the visual fields using only a paper test chart, a record sheet, and a pencil. This method,

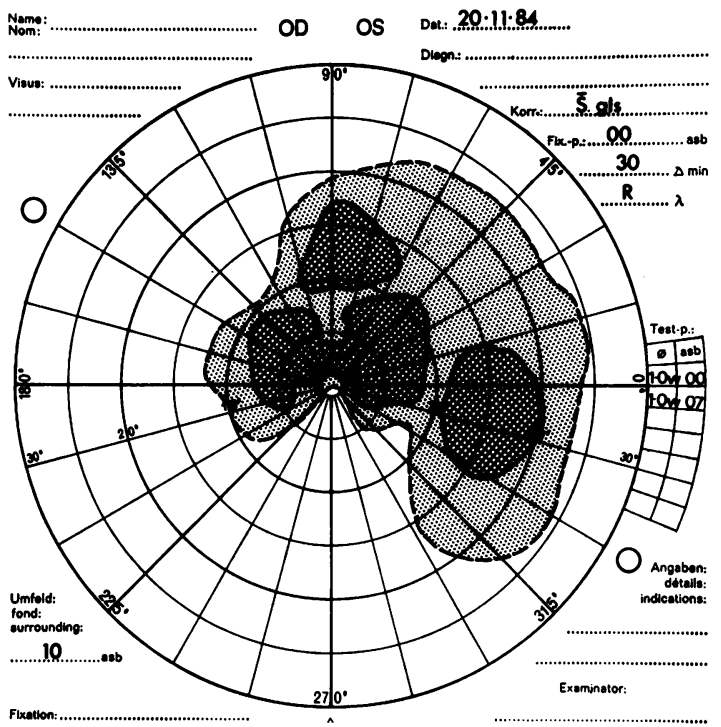

Fig. 3A

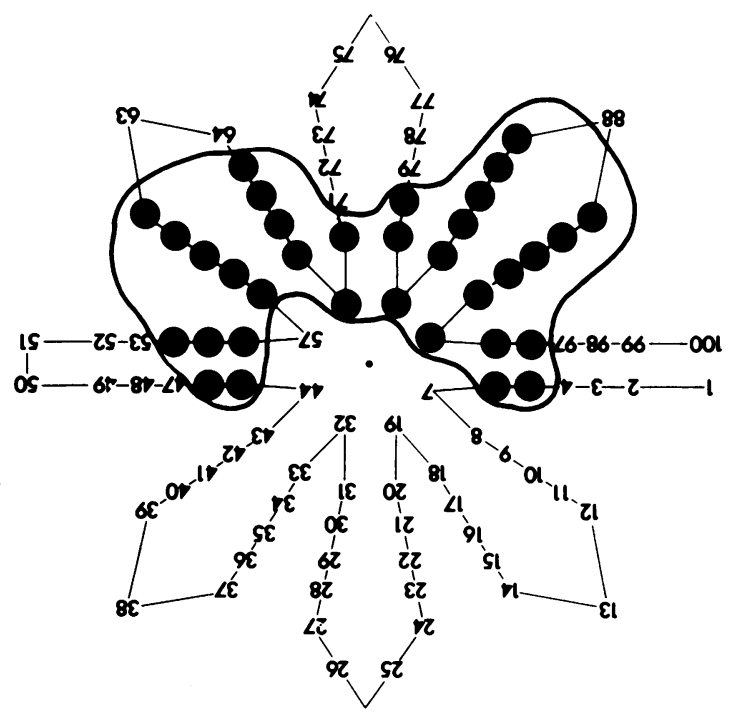

Fig. 3C entitled 'oculokinetic perimetry', might facilitate the detection and management of glaucoma, especially in situations where conventional perimetry is not possible. Susceptible individuals who cannot receive adequate supervision because they live in remote areas could use disposable test charts to perform

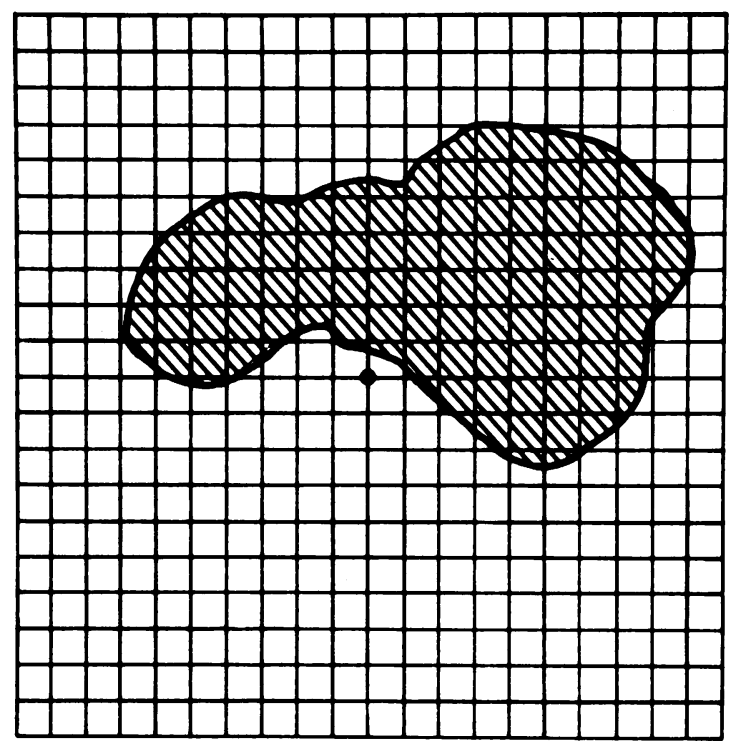

Fig. 3B

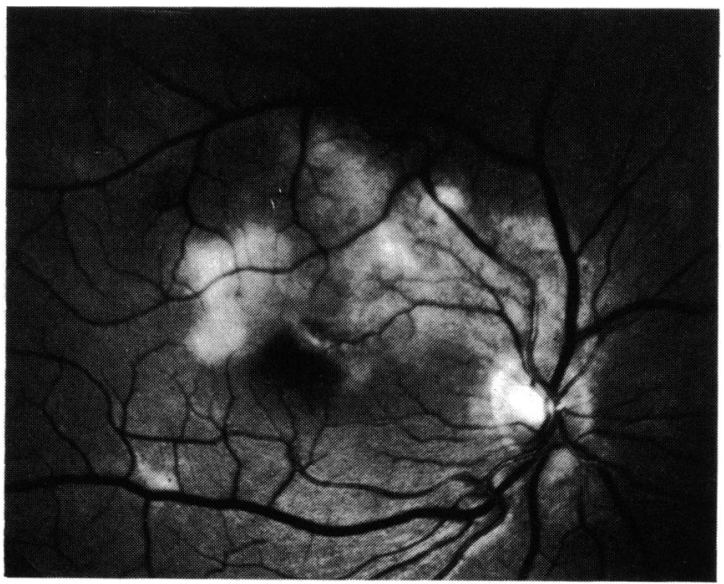

Fig. 3D

Fig. 3 The right central visual field of a 48-year-old female suffering from serpiginous choroiditis plotted (A) with the Tubinger perimeter, (B) by means of the Amsler chart, and (C) by oculokinetic perimetry. The last test was performed with the patient standing and the chart at knee level, and an adhesive red disc was used as the test stimulus. (D) $A$ black and white photograph of the right fundus which has been inverted and reversed to facilitate comparison with the plotted visual fields. 


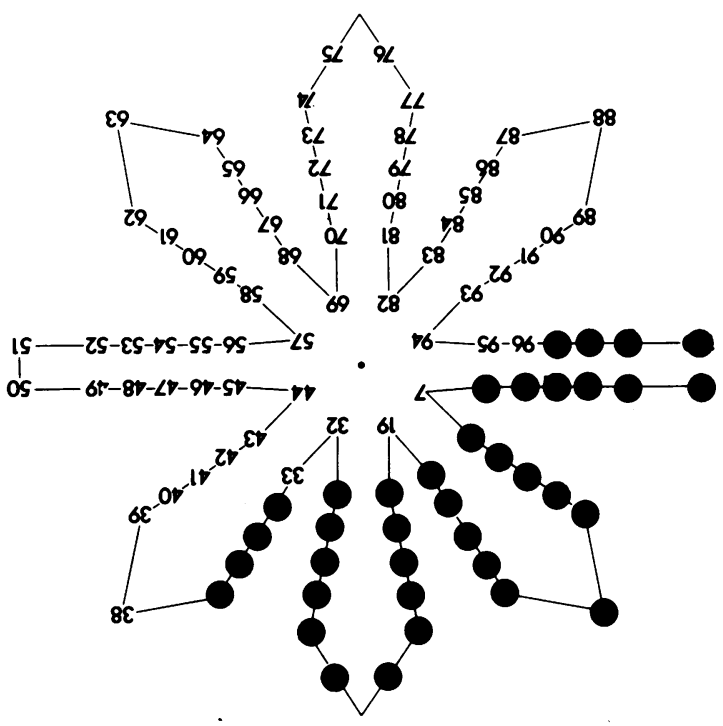

Fig. 4A

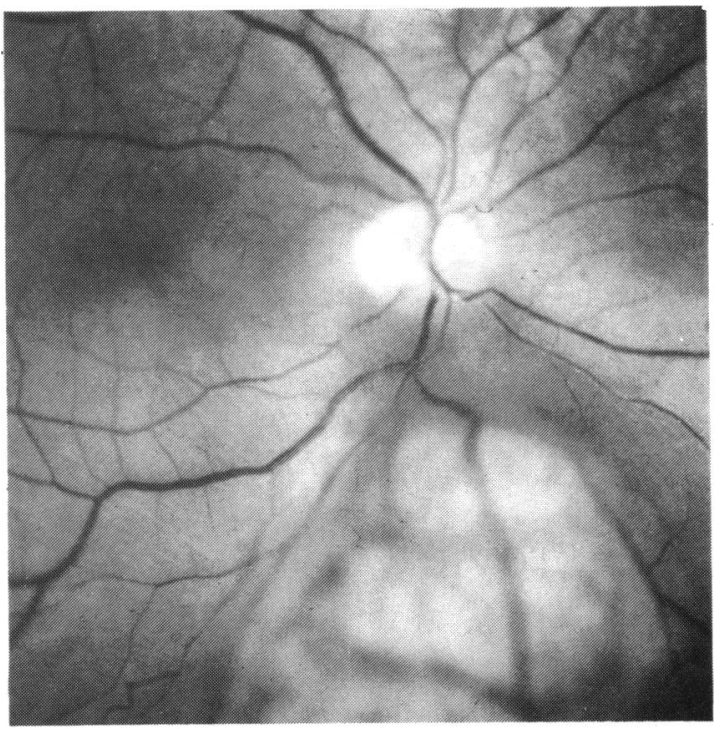

Fig. 4C

periodic self-assessment at home with the assistance of a friend or relative if necessary. Larger and more convenient oculokinetic visual field test charts would enable non-ophthalmic health care workers to participate in screening programmes for glaucoma, especially in underdeveloped countries. It must be emphasised that the use of this test as a convenient substitute for more reliable conventional perimetry is not recommended.

Oculokinetic perimetry could be of benefit to patients whose vision is threatened by other diseases

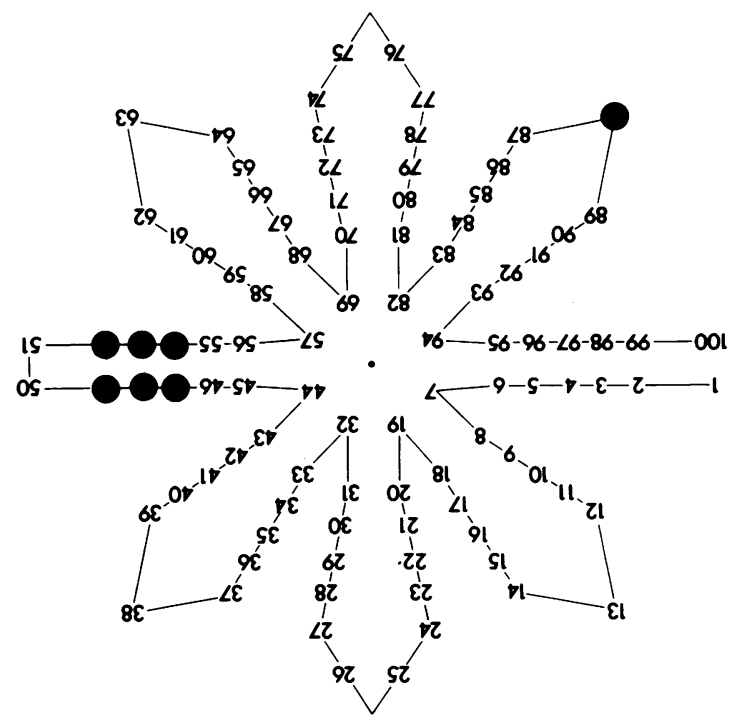

Fig. 4B

Fig. 4 The central visual fields of a 46-year-old female plotted by oculokinetic perimetry to show (A) an inferotemporal defect in the right eye and (B) a normal visual field in the left eye. (C) a black and white fundus photograph of the right eye, showing a superonasal choroidal melanoma, which has been inverted and reversed to facilitate comparison with the plotted visual field.

as well as glaucoma (Figs. $3 \mathrm{~A}, \mathrm{~B}, \mathrm{C}, \mathrm{D}$, and $4 \mathrm{~A}$, $B, C)$. For maximum efficiency special charts would have to be designed for each specific purpose. In areas where innumeracy might be common the test would have to be modified so that the subject's gaze was directed across the chart by means of a series of arrows instead of numbers.

Research is still in progress and it is hoped that results will eventually be published demonstrating the precise scope of oculokinetic perimetry and the most efficient means of its deployment.

I am grateful to Professor W S Foulds, Dr J L Jay, Dr D Allan, Dr G N Dutton, Dr S B Murray, and Miss E McClure for their helpful advice; Mrs A Currie and the Medical Illustration Department for the art work; Miss A McFadden for secretarial assistance; and to the opticians, nurses, and volunteers who carried out the test. The Visual Research Trust, Glasgow, funded the study.

\section{References}

1 Harrington DO. The visual fields: a textbook and atlas of clinical perimetry. 5th ed. St Louis: Mosby, 1981.

2 Heijl A. Computerised perimetry. Trans Ophthalmol Soc UK 1985; 104: 76-87.

3 Amsler M. Earliest symptoms of disease of the macula. $\mathrm{Br} J$ Ophthalmol 1953; 37: 521-37.

4 Flocks M. Visual screening via television. Surv Ophthalmol 1983; 28: $184-7$. 